\title{
An observational study of cross-cultural communication in short-term, diverse professional learning groups
}

\section{Background}

For 3 weeks during August 2013, the University of Salford hosted a unique summer school programme for students and qualified radiographers, psychologists and physicists. More than sixty students and tutors from the UK, Switzerland, Norway, Portugal and the Netherlands participated (see editorial for this special edition). The programme comprised six multicultural groups each of which was required to plan and conduct an experiment related to x-ray dose and image quality and then write up their work as an article for journal submission. The project was funded through the European Commission's Erasmus Intensive programme

Erasmus is part of the European Commission's scheme for promoting international study. The aim is to increase student mobility within the European Community ${ }^{1}$ with the long term goal of promoting and enabling globalisation of the workforce. The Intensive Programme provides opportunities for Higher and Further Education students, teachers and institutions to work together over short periods of time, typically 3-6 weeks, to satisfy 3 objectives $^{2}$. The study being reported here is concerned with the evaluation of the second objective which is to:

"Enable students and teachers to work together in multinational groups and so benefit from special learning and teaching conditions not available in a single institution, and to gain new perspectives on the topic being studied"1;

The study therefore aims to explore whether students and teachers can work together in multicultural groups in order to realise these benefits or whether cultural differences have the potential to hamper the effectiveness of the group.

\section{Literature review}

There has been much written on multicultural groups and the factors which can influence their performance. Many believe that collaboration benefits from diversity ${ }^{3-6}$ as this promotes consideration of others' perspectives, and the more diverse these perspectives are, 
the richer the learning experience. Consequently it has been shown that diverse groups produce higher quality ideas than homogeneous groups ${ }^{7}$.

However, research comparing culturally homogeneous and heterogeneous groups in terms of productivity, or outputs, shows there are circumstances in which diverse groups can be less effective ${ }^{8-10}$. It has been suggested that this is because the benefits of diverse opinions are not always realised due to failure in communication ${ }^{11,12}$. These concerns relate not only to semantics i.e., language use, but to socio-pragmatic failure. Socio-pragmatics is concerned with the way people interpret meaning based on the social and cultural context in which the communication takes place. As such, because interpretations are culturally-bound, when two people from diverse cultures interact there is the potential for misinterpretation, even when a common lexicon is employed.

However, it is believed that over time adaptation to another's communication strategy occurs, enabling diverse groups to perform just as well. Watson, Kumar et $\mathrm{al}^{13}$ compared culturally diverse and homogeneous student groups for task performance and showed that whilst homogeneous groups performed better initially, after 17 weeks the difference in performance between the two groups was not significant.

Because the Erasmus Intensive Programme was only 3 weeks in length we were interested to know whether this would be sufficient time for the work groups to adapt in terms of communication or if socio-pragmatic failure might hinder progress and output. We therefore needed to employ a suitable tool for analysing these concerns.

\section{Rapport Management theory}

Rapport Management (RM) is a framework of cross-cultural communication ${ }^{14}$. It suggests the interactants (people involved in a communicative interaction) have three competing concerns during interaction i) face, ii) sociality rights and obligations and iii) interactional goal. Interactants balance these concerns through tacit communication strategies and what motivates them to prioritise one base over another is influenced by culture and context.

'Face' as described by Goffman (1967) ${ }^{15}$ is a sociological concept related to esteem, worth and dignity and is what is claimed/ protected by a person in a communicative act. However, face is both a social and a dynamic concept in that it is constructed in interaction and is 
determined not only by one's self-belief of what is 'face-worthy' but also by the judgement of others in the interaction. Therefore, what is worthy of approval in terms of face is dependent on many contextual factors including the perspective of each individual and the influence of wider culturally-related beliefs. A 'face-threatening act' (FTA) is experienced when a speaker makes a move which puts themselves or the hearer at risk of face loss, and like face-worthiness, what constitutes 'face loss' is also culturally bound. The RM framework further divides face into two categories: 'quality face' and 'social identify face'. The former is related to our need to be positively valued by others in terms of personal qualities. Whereas social identity face is concerned with how we are valued in the social roles we perform, i.e., our sense of public worth and is therefore particularly relevant when studying groups.

'Sociality rights' are concerned with our perceived entitlements and obligations in relationships with others. Such rights include i) 'equity' in relationships: related to a mutual understanding that there should be a balance in demands made on each other's resources, and ii) 'association', which clarifies the level of 'involvement' versus 'detachment' expected in an interaction. This can be considered in terms of either involvement in the task emotional involvement with the other interactant/s.

In addition to face and sociality rights is the "interactional goal ${ }^{12}$, that is, the function or purpose of the interaction. Thus RM acknowledges the relevance of task achievement in maintaining relations since a mismatch between the importance each interactant places on the goal can cause a breakdown in communication. Linguistically, the interactional goal refers to the purpose of each individual utterance. For the purpose of this study we shall take interactional goal to be the goal or task overall, i.e. the purpose for which the group was configured.

Thus in the RM framework, face, sociality rights and interactional goal form the three main bases of communication, and it is posited that people in communicative interactions are constantly evaluating their moves according to the relative importance of these three concepts. Each person in the interaction may weigh the relative importance of the RM bases differently as a result of the influence of their own cultural and social background. In communication with someone from a different culture, what is taken as acceptable and what 
constitutes loss of face may not always be clear or shared. The RM framework is therefore an ideal tool for analysing culturally diverse groups.

The aim of this research was therefore to explore the influence cultural diversity might have on group collaboration and learning goal. It was not the intention to compare one national or ethnic group with another. Rather this was an exploratory study that aimed to identify whether concerns with managing rapport could interfere with task completion, and thereby to gain insight into how such groups of students might be supported. The questions which framed this research were:

- In a small intercultural learning group whose task time is limited, what motivates Rapport Management?

- Are these motivational forces influenced by changes in the group over the short time they are together?

- How can tutors facilitating short-term intercultural learning groups manage potential barriers to learning?

\section{Method}

Types of data

i) Observational data: One group of 7 students and 2 tutors was observed over two 2hour sessions; the first at the beginning of the project the second was during the final day of group work. There were 10 days between these two sessions, but students worked and socialised together every day during this time.

All groups were asked if they wanted to participate and only those groups where all members consented would be considered. However, in only one group did all students and tutors consent to take part. This became the study group. The demographics of the group can be found in table 1 . There were 7 students, 3 from the UK, 2 from Portugal, 1 from Switzerland and 1 from the Netherlands. There were 2 tutors, 1 from Switzerland and 1 from Portugal.

$<<$ table 1 here $>>$ 
Observed interactions were captured using video camera. These interactions were not transcribed but were analysed directly from the video to enable replay for clarification purposes (sample extracts were transcribed for illustration purposes found later in this paper). RM was used as the analytical framework to identify the motivational force for communication, in other words whether face, sociality rights or interactional goal predominated, and whether these motivational forces changed between the two sessions.

ii) Interview data: Two of the students were interviewed for verification of researcher interpretations. Spencer Oatey (2002) ${ }^{14}$ explains that it is the interpretation of an utterance by the people involved in an interaction which renders an utterance facethreatening, as no utterances are intrinsically face-threatening. Therefore triangulating student and researcher interpretations in this was way was important for validating the results. This task of verification requires clarification and confirmation of contextual issues not captured on video. The students were therefore interviewed together rather than individually to maximise memory of the context.

Selection of students for the interview was determined by convenience as it was necessary to interview students after analysis of the observational data. By this time the students had returned to their respective countries therefore it was only possible to interview UK students. Telephone interviews would have been difficult since students were required to view video clips during the interview.

Interview responses were collected as researcher notes. These were not analysed using a specific framework but were used to clarify and add verification to interpretations of the observational data.

\section{Task}

The group's task was to design and conduct an experiment to compare dose and image quality. The first week's activities were primarily individualistic, comprising didactic lectures related to underpinning knowledge. Weeks 2 and 3 were group-based and involved discussions and data collection activities. Tutors were expected to support the students in achieving their outputs. They were provided with a tutor handbook and were briefed daily 
about methodological issues related to the experiments, but were not given specific guidance about facilitating groups.

Ethical approval was obtained from the University of Salford Research, Innovation and Academic Engagement Ethical Approval Panel (HSCR13/39). All participants provided written consent and were assured that anonymity would be preserved. Pseudonyms have been used in the examples below.

\section{Results}

Table 2 summarises the emphasis placed on each of the RM bases by the group and how this changed between session 1 and session 2 . The following will describe each session in more detail with reference to the RM bases.

$<<$ table 2 here >>

\section{Session 1 (2 hours, beginning week 2)}

The first recording took place in a classroom with students initially sitting in one group around a large table. The purpose of this session was for the group to design their experiment and agree how this would be conducted. The session started with the Erasmus Programme Manager (a third tutor with overall responsibility for the summer school) outlining the task to the group and clarifying concerns. The students were then left to carry on the discussion under tutor supervision. Approximately half way through the session the students began to work in small groups. There was no obvious catalyst for this tactic. In the last 20 minutes the students returned to their original places. One student was clarifying a task with the tutors separate from the main group. The rest of the students carried on talking quietly in pairs. Conversation in this last 20 minutes mainly concerned task clarification for the next day. Two students were heard to be discussing social arrangements for the evening.

\section{Face \\ Quality face}

There was evidence to suggest up-holding quality face of self and other was the prime concern in session 1. This manifested in Face Threatening Act (FTA) avoidance. In the following example (1 hour into the session), two students from different countries are working together. One (Ben) uses English as a first language and the other (Eli) does not. The two students are discussing the notion of 
subjectivity in film appraisal. Ben is typing the discussion onto a laptop (numbers in brackets are pauses in seconds, square brackets are over-talking and double brackets are non-verbal actions ${ }^{16}$ ).

At line 6, Eli hesitatingly agreed with Ben although what follows shows she did not agree. She began to contradict, (which is regarded as an FTA) ${ }^{17}$ and decided to avoid this act at line 8 dismissing her interjection. This suggests she considered the threat to Ben's quality face and the threat to her own, in having to explain a tricky concept in a second language, too great and so chose an avoidance tactic instead.

1. Ben and again it's perceptual image quality cos we're using observers

2. Eli [[yeah]]

3. Ben [[it's either]] perceptual or obj...subjective

4. Eli ((thoughtful with hand on chin stroking mouth with forefinger, nods))

5. Ben cos we'd have to specify if it's objective or subjective (2) so it's subjective

6. Eli it's subjective (1.5) yeah ((Ben types on laptop))

7. (4)

8. Eli but (2) no ((dismisses her comment with shake of hand-deciding not to go on))

Other FTA avoidance strategies included students not answering tutor questions, prolonged silences and individualistic activities such as some students clarifying tasks with the tutor in 'aside' conversations rather than with the rest of the group; that is talking through the tutor rather than directly to the others. There were two (UK) students who were more likely to speak out and they tended to talk to each other rather than the group. There was one Portuguese student who was also more likely to respond to tutor questions. Interview data confirmed that some of the very quiet students were lacking in confidence with their English speaking skills and the UK students admitted not wanting to expose these students' lack of English, thus showing consideration for their quality face.

\section{Social Identity Face}

There was little evidence of concerns for social identity face. Nobody appeared to be acting out a social role in the group such as leader, secretary etc. One student was seen to ask the tutor in a quiet voice whether the group should talk about task allocation for the following day. For this student, the need to establish social identity face was subordinated to the need not to threaten personal quality face (i.e wanting to 
lead but being seen by others as being 'pushy'). Interview data confirmed no roles had been defined or allocated although at various stages of the project tasks had been ascribed.

Tutors displayed behaviours typically associated with the tutor role. When students wanted some guidance with their task they would ask the tutor who would join them in their small group and answer the specific problem. Tutors also took the lead in directing discussions about the task and posing questions to the group. In this respect, the social identity face of the tutors was maintained. Interviews with the students confirmed that they thought the tutors were very effective.

\section{Sociality rights}

\section{Association/Disassociation}

In terms of 'emotional' involvement, students favoured disassociation rather than association. For example, at times when the tutor were attending to individual students the rest of the group was silent (the longest silence of which was 14 minutes) i.e. there was little off-task discussion, and at one point one student was surreptitiously eating biscuits without sharing them. There was little evidence of warmth, humour or laughter initially, although this developed over the course of the session, especially following the small group work. This contradicts the interview data where the students believed the group had a strong social dynamic, however the interview took place several weeks after the end of the summer school so the students' perception of group rapport during the first session may have been influenced by the passage of time during which the students had engaged in social intercourse.

In terms of involvement in the task, some students demonstrated more participation than others. It is not clear what motivated this but the ones who were less involved appeared to be those who had less well-developed English skills. Lack of involvement may therefore have been related to the protection of quality face, which would have been threatened had the students been required to engage in cognitively difficult conversations that demand good spoken English. 


\section{Equity: Autonomy/imposition}

The preference was for students to retain autonomy in interactions rather than to be imposed upon, or to impose on others. There were very few occasions where requests were made of one another. Rather, it was the tutors who were making requests of individual students. There was no evidence that the group had formalised the concept of sociality rights through a set of ground rules or principles which would have made explicit the expected level of participation, and the acceptability of requests and challenges for getting the job done.

\section{Interactional Goal}

There was a strong emphasis on the interactional goal/group task. The majority of interactions focussed towards this rather than off-task social discussion. However, despite the importance of the task for the group, the overarching theme for this first session was not to threaten quality face. Motivation for the choice of communicative strategies was therefore the preservation of quality face which had the potential to leave a task undone and therefore negatively influence the productivity of the group at this planning stage. An example of this could be seen towards the end of the session where one student raised a complex question regarding data collection points with the tutors. The rest of the students remained silent rather than joining in to help solve the problem and this silence persisted for 14 minutes.

\section{Session 2 (2 hours, end of week 3 )}

The second recording also took place in a classroom. The purpose of the session was for the students to write a joint report. Two high-involvement students sat on the back row with one of the tutors and were involved in a separate task related to the report. They talked between themselves. The rest of the students and the remaining tutor faced the front of the classroom, seated in rows rather than around a table. The report was projected onto a screen whilst one student typed up the report as it was being dictated to her. The dictation was carried out by the third (Portuguese) high-involvement student and the second tutor. The other three students were sitting around the periphery of the classroom and spoke very little during the session. 


\section{Face}

Quality Face

Compared to session 1, there were more instances of threat to quality face. However, these threats were only undertaken by high-involvement students and tended to be directed at the other high-involvement students. In this example, Piri had been leading the dictation of the report, English was not her first language and she was tiring (she was heard to say she needed inspiration and kept lapsing into Portuguese). The other high-involvement students were not actively involved in dictating the report as they were completing a data analysis task. They were sitting behind Piri. The low-involvement students were not providing Piri with any help:

1. Piri ((sighing and turning towards the back row)) shall we all look at this discussion

2. Ben sorry I apologise

3. Piri that's ok

Even though she emphasised the word 'all', Piri was looking for help only from the high-involvement students (she turned to face these). The low-involvement students' quality faces were therefore maintained, whilst the quality face of the highinvolvement students was threatened.

\section{Social Identify Face}

As with session 1, there was still little evidence of concerns for social identity face because nobody had been formally allocated to a role. However, informally, role allocation was apparent. For instance, in the example above, Piri made demands of the high-involvement students and in this way she was implying they were not upholding their social identity face, but she did not make the same demands of the low involvement students. By apologising (line 2) Ben's acknowledged this expectation and his failure in upholding his social identity face. Consequently there appeared to be an acceptance by the group that the low-involvement students were not expected to contribute, so they were not motivated by a need to uphold their social identity face. This is evidenced in the fact there were no apologies from them or any offers of help.

\section{Sociality rights}

Association/Disassociation 
Compared to session 1, there were more signs of affective involvement from the high-involvement students in the form of banter and joking. In this example the tutor asked Ged (a high-involvement student) whether he had finished typing up the results from the work produced by the other students

1. Tutor you have finished the results?

2. Ged no I didn't understand Portuguese so I couldn't

3. Tutor oh no, ((laughter))

4. (2)

5. Piri Google translator

6. ((Laughter from all group except two of the low-involvement students))

7. (2)

8. Ged too much effort

Ged's response is to joke about Piri's written English, suggesting it was still in need of translation. Piri and Ged are comfortable with the banter as illustrated with the laughter. During banter between the high-involvement students the low-involvement students did not join in but smiled. Furthermore, the low-involvement students did not engage in conversation so it was difficult to identify their emotional involvement with the others and whether this had changed between the two sessions.

In terms of task involvement there appeared to be two distinct groups of students. One group comprised the three high-involvement students who tended to lead the discussion and manage the tasks. They made requests and issued instructions to one another. The low-involvement group generally observed; they appeared to be listening and were not disruptive but they did not communicate verbally and they were seen to be yawning and less-animated in their body language than the highinvolvement students. The student who was typing was involved in the task but was not involved in deciding what should be written, she was following instructions only.

\section{Equity: Autonomy/Imposition}

As indicated above, the low-involvement students were generally not imposed upon by the high-involvement students, however as identified earlier high-involvement students expected to be imposed upon and apologised if found wanting in this regard. 


\section{Interactional Goal}

In this session, the interactional goal or group task appeared to be motivational driver for the communicative strategy. The group was very conscious of the deadline and this led to some face-threatening moves. In the following example, Piri was giving directions to Ida who was typing up the report.

1. Piri so er ((pointing at screen)) when it says there continue like er almost in last paragraph continue it means that no $\underline{\text { no }} \underline{\text { no }} \underline{\text { down }} \underline{\text { down }} \underline{\text { down }}$ ((instructing Ida

2. (1) to scroll down the screen)

3. Ida sorry? (turning round to face her)

4. Piri when it says like there continue it means that you can put the erm that explanation you give it in the first "the possible explanation for this is the variable"

5. Ida this one there?

6. Piri yes because I explained that but the explanation comes afterwards

Piri used direct speech without any politeness speech modifiers but with emphasis and repetition. This can appear abrupt and face-threatening ${ }^{17}$. Although it is possible that Piri generally uses a more direct speech in normal speech this is not evident when comparing data from both sessions, furthermore, later in the session she justified dictating the report in a non-consensual manner to the tutor with; "I'm just saying... so we can hurry up". Student interview data confirmed that it was the pressure of the imminent deadline for the interactional goal which motivated a more direct communication strategy.

However, the interactional goal was not sufficient for low-participation students to risk threatening quality face but may have been the reason why they allowed the high-participation students to dominate and lead - they too wanted to see the task completed.

\section{Interview data}

This data was used to validate interpretations and provide further information about the group dynamic off-camera. The students reported that there was a great deal of social interaction between the members of the group outside of the recorded sessions. They had engaged in social activities (eating out, going to the pub) most nights of the programme and used on-line social networks on a regular basis which persisted at the time of the interview; 
several months following the end of the project. The students described the group as very friendly and close. This appeared to contradict the observations.

When the two students were shown episodes of face threat, instigated by themselves in session 2, they were surprised and had not anticipated that their interactions would impinge on the quality face of others. They did not feel that these moves had been interpreted negatively by the other students either. They cited their continuing social networking as evidence of this view. However, they also stated that the pressure of finishing the task had driven them to make such interactional moves.

They confirmed the researcher interpretations that they had respected the sociality rights of the low-involvement students, and not impinged on their desire for autonomy, citing two reasons for this: i) some of them had shown themselves to be incapable of the task (i.e. taskspecific reasons), ii) some of them had been experiencing personal difficulties (i.e. social reasons).

\section{Discussion}

The discussion will consider the three questions posed at the beginning of the study.

In the first session, this group was primarily motivated by concerns for quality face. The interactional goal was not sufficient to overcome the preference for students to protect quality face and avoid face threat. English as a second language appeared to be one of the major threats to quality face. Although this did not present barriers to communicative understanding, analysis using the RM framework showed that it influenced motivation for communication. This is because it was responsible for threatening the quality face of those students who lacked confidence in spoken English which in turn led to low-involvement, putting group productivity at risk. Students who had good spoken English were more likely to participate.

At the second session the interactional goal did drive face-threatening moves by the more dominant students, but these students were still sensitive to, and respected, the autonomy of the low-involvement students. Therefore, affective association, facilitated through the social activities arranged by the group, was implicit in the respect and sensitivity students showed to one another. It was reported in the early literature on face management that a reducing 
social distance (i.e. interactants becoming better acquainted socially) lessens the impact of face-threatening moves ${ }^{17}$ so social interaction is also likely to have mitigated the FTAs between high-involvement students witnessed in the second session. Therefore a change in communication strategy between the 2 recorded sessions was apparent and appeared to be motivated by the interactional goal but, importantly, this was tolerated as a result of an enhanced emotional attachment.

Throughout the project there was no evidence of concern for social identity face (i.e. sense of public worth) and this may be because none of the students was explicitly allocated to a particular role at the beginning of the project. Furthermore there were no ground rules which, along with allocated roles, would have legitimised face threat. In other words, an allocated leader upholding their social identity face would have been expected to encourage a more equal participation, and the low-involvement students may have felt obligated to contribute. This would in turn have led to improved group efficiency rather than a polarisation of high- and low-involvement groups where some members' skills and opinions were not utilised. The importance of group ground rules to address these issues has been extensively explored and is widely advocated in the group learning literature ${ }^{11,18,19}$. However, for this group an unequal involvement did not lead to tensions despite a lack of rules and formalised roles because as mentioned previously socialisation outside the classroom appears to have led to a greater understanding between members.

The tutors were seen to uphold their own social identity face based on traditional assumptions of the role of the tutor. For instance, in session one, they managed the discussion, answered questions and directed and allocated tasks. In session two, the tutors were also involved in task-specific activities. The students reported that they were effective tutors. However, the tutors did not appear to give the same level of priority to the facilitation of group dynamics. For example, the tutors did not take steps to encourage the participation of low-involvement students. Lack of attention to supporting the group dynamic has been shown to have a negative impact on student motivation ${ }^{20}$. Tutors moving from traditional modes of teaching to more problem/project-based approaches require additional skills and a changed perspective of their role in order to facilitate effective student participation ${ }^{21,22}$. The tutor role therefore needs clarification and tutors may need support and training in how to manage group dynamics to ensure the task is not put at risk and that all students can benefit from participation. 
There were a number of limitations to this study. The video was analysed by one (UK) researcher whose interpretation of the data is likely to have been more heavily influenced by her own cultural background. However student verification helped to support interpretations and explain the social development of the group outside the observed sessions. The group was self-selecting nevertheless the issues highlighted were not intended to be a generalisation of what would happen in all groups but an exploration of the sorts of considerations that might need to be made. Interviews with low-involvement students and tutors were not possible but could be included in evaluation of the next summer school. Observational data of any kind is subject to participant reactivity, where those being

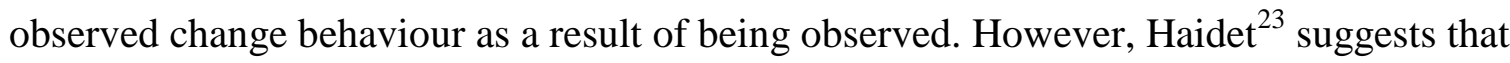
this is minimised following the initial 5 minutes of observation. Analysis of the two 2-hour videos took this into consideration.

\section{Conclusion and recommendations}

Using the RM framework, this project explored whether objective 2 of the Erasmus Intensive summer school was achievable. It is clear that there are no cultural barriers to success for such groups and providing tutors are equipped with the skills and knowledge to support multicultural groups, new perspectives can be gained; objective two of the Erasmus Intensive Programme is therefore certainly achievable.

However the results also highlight that in order for tutors and students to work together there needs to be a clear set of roles and ground rules so that the entire group are supported in managing short term projects. Socialisation is also crucially important to enable face threatening moves, (inevitable when task deadlines are short and imminent) to be tolerated. English language skills also need some consideration. Where students are not confident in these they will need support to ensure they do not become marginalised.

\section{Conflict of interest statement}

The authors have no conflicts of interest to declare. 


\section{References}

1. British Council Erasmus [online] http://www.britishcouncil.org/erasmus-background.htm [accessed 21/1/14]

2. British Council. IPSOS Public affairs, Boos, Allen, Hamilton. Culture AT WORK The value of intercultural skills in the workplace [online] http://www.britishcouncil.org/culture-at-workresearch_march_2013.pdf 2013 [accessed 21/1/14]

3. Taylor I, Burgess H. Responding to 'Non-traditional' Students: An Enquiry and Action Approach. The Challenge of Problem-based Learning. D. Boud and G. Feletti. London, Kogan 1997; Page: 103-16.

4. McLean MJ, van Wyk JM, Peters-Futre EM, Higgins-Opits SB. The small group in problembased learning: more than a cognitive 'learning' experience for first-year medical students in a diverse population. Medical Teacher 2006; 28(4): E94-E103.

5. Mills PC, Woodall PF, Bellingham M, Noad M, Lloyd S. Using group learning to promote integration and cooperative learning between Asian and Australian second-year veterinary science students. Journal of Veterinary Medical Education 2007; 34(3): 350-5.

6. Singaram VS, Dolmans DHJM, Lachman N, van der Vleuten CPM. Perceptions of Problem Based Learning (PBL) Group Effectiveness in a Socially-Culturally Diverse Medical Student Population. Education for Health 2008; 21(2): 1-9.

7. Oetzel JG. Self-Construals, Communication Processes, and Group Outcomes in Homogeneous and Heterogeneous Groups. Small Group Research 2001; 32(1): 19-54.

8. Chatman JA, Polzer JT, Barsade SG, Neale MA. Being Different Yet Feeling Similar: The Influence of Demographic Composition and Organizational Culture on Work Processes and Outcomes. Administrative Science Quarterly 1998;43(4): 749-80.

9. Pelled LH, Eisenhardt KM, Xin KR. Exploring the black box: An analysis of work group diversity, conflict, and performance. Administrative Science Quarterly 1999; 44(1): 1-28

10. Singaram VS, van der Vleuten CPM, Stevens F, Dolmans DHJM."For most of us Africans, we don't just speak": a qualitative investigation into collaborative heterogeneous PBL group learning. Advances in Health Sciences Education: 2010; 1-14. 
11. Robinson L. The influence of cultural diversity on student learning interactions: a qualitative study of rapport management in an undergraduate problem based learning group. Huddersfield, University of Huddersfield. EdD Thesis: 2011, p. 1-250.

12. Spencer-Oatey H. Ed. Culturally Speaking: Culture, Communication and Politeness. London, Continuum; 2008, p.11-47

13. Watson WE, Kumar K, Michaelsen LK. Cultural Diversity's Impact on Interaction Process and Performance: Comparing Homogeneous and Diverse Task Groups. The Academy of Management Journal 1993 36(3): 590-602.

14. Spencer-Oatey H. Managing rapport in talk: Using rapport sensitive incidents to explore the motivational concerns underlying the management of relations. Journal of Pragmatics 2002; 34(5): $529-45$.

15. Goffman E. Interaction Ritual. Essays on Face-to-Face Behaviour. New York, Pantheon;1967

16. Sacks H, Schegloff EA, Jefferson G. A simplistic systematics for the organisation of turn-taking for conversation. Language 1974; 50(4): 696-735

17. Brown P, Levinson SC. Politeness: Some universals in language use. Cambridge, UK, Cambridge University Press; 1987

18. Maudsley G. Do we all mean the same thing by "problem-based learning"? A review of the concepts and a formulation of the ground rules. Acad Med 1999; 74(2): 178-85.

19. Azer SA. Becoming a student in a PBL course: twelve tips for successful group discussion. Medical Teacher 2004; 26(1):12-15.

20. Mete S, Yildirim Sari H. Nursing students' expectations from tutors in PBL and effects of tutors' behaviour on nursing students. Nurse Education Today 2008; 28(4): 434-42.

21. Northedge A. Enabling Participation in Academic Discourse. Teaching in Higher Education 2003; 8(2): 169-80

22. Moust JHC, van Berkel HJM, Schmidt HG. Signs of erosion: Reflections on three decades of problem-based learning at Maastricht University. Higher Education 2005; 50(4): 665-83.

23. Haidet KK, Tate J, Divirgilio-Thomas D, Kolanowski A, Happ MB. Methods to improve reliability of video-recorded behavioural data. Research in Nursing \& Health 2009; 32(4): 465-74 
